\title{
Mandenkan
}

MANDENIKAN Bulletin semestriel d'études linguistiques mandé

$59 \mid 2018$

Numéro 59

\section{La catégorie des verbes statifs dans le système morphosyntaxique du soninké}

Stative verbs in the morphosyntactic system of Soninke

СТАТИВНЫЕ ГЛАГОЛЫ В МОРФОСИНТАКСИЧЕСКОЙ СИСТЕМЕ СОНИНКЕ

\section{Denis Creissels}

\section{OpenEdition}

Journals

Édition électronique

URL : https://journals.openedition.org/mandenkan/1271

DOI : 10.4000/mandenkan. 1271

ISSN : 2104-371X

\section{Éditeur}

Llacan UMR 8135 CNRS/Inalco

Référence électronique

Denis Creissels, "La catégorie des verbes statifs dans le système morphosyntaxique du soninké », Mandenkan [En ligne], 59 | 2018, mis en ligne le 20 juillet 2018, consulté le 08 juillet 2021. URL : http:// journals.openedition.org/mandenkan/1271 ; DOI : https://doi.org/10.4000/mandenkan.1271

Ce document a été généré automatiquement le 8 juillet 2021.

\section{c) (†)}

Les contenus de Mandenkan sont mis à disposition selon les termes de la Licence Creative Commons Attribution - Pas d'Utilisation Commerciale - Partage dans les Mêmes Conditions 4.0 International. 


\section{La catégorie des verbes statifs dans le système morphosyntaxique du soninké}

Stative verbs in the morphosyntactic system of Soninke

СТАТИВНЫЕ ГЛАГОЛЫ В МОРФОСИНТАКСИЧЕСКОЙ СИСТЕМЕ СОНИНКЕ

Denis Creissels

\section{Introduction}

1 Le soninké possède une classe de lexèmes dont les caractéristiques morphosyntaxiques sont comparables à celles des verbes statifs des langues mandingues. ${ }^{1}$ Comme son analogue dans les langues mandingues, la classe des verbes statifs du soninké regroupe quelques dizaines de lexèmes (j'en recense une trentaine dans cet article, et il y en a vraisemblablement d'autres), et les signifiés lexicaux des verbes statifs du soninké sont du même type que ceux des verbes statifs du mandingue. Or cette catégorie passe pratiquement inaperçue dans les deux grammaires de référence du soninké disponibles jusqu'ici. La grammaire de Yacouba Diagana (1994:98-101) comporte seulement une brève mention de trois verbes statifs qui ont la particularité d'avoir un emploi d'auxiliaire aspectuel ou modal (gàbá 'être fréquent', qáwà 'devoir' et síri 'devoir'), et mentionne comme une simple bizarrerie leur combinaison avec les marqueurs prédicatifs nàn et ntá (alors qu'il s'agit là précisément du critère d'appartenance à la catégorie des verbes statifs). Quant à Ousmane Moussa Diagana (1995:263-264), il est clair qu'il a entrevu l'existence de cette catégorie mais n'a pas tiré toutes les conséquences de ses observations, qui restent d'ailleurs incomplètes (du fait notamment qu'il ne mentionne pas l'existence d'une forme négative des verbes statifs).

2 L'objectif de cet article est donc essentiellement d'attirer l'attention sur la nécessité de prendre en compte cette catégorie dans la systématique des parties du discours du soninké. 
3 Les données traitées dans cet article sont tirées de l'enquête lexicale qui a fourni le corpus pour l'analyse phonologique du soninké du Kingi (Creissels 2016). Autrement dit, ces données peuvent avoir été inspirées par divers documents publiés, notamment les dictionnaires: (Diagana 2011; Smeltzer \& Smeltzer 2001), mais dans tous les cas elles ont été vérifiées par un consultant locuteur natif du parler du Kingi, et le cas échéant adaptées selon ses indications. Je ne fais donc aucune hypothèse quant à la représentativité de ce qui est décrit ici relativement aux autres variétés du soninké. Je n'ai aucun indice qui tendrait à suggérer l'existence de variations importantes pour cet aspect particulier de la morphosyntaxe du soninké, mais je ne dispose pas non plus des données qui permettraient de dire précisément jusqu'à quel point ce qui est décrit ici s'appliquerait ou non aux autres parlers. En toute rigueur, dans la suite de cet article «le soninké » doit être compris comme une abréviation pour «les variétés de soninké qui fonctionnent comme celle que j'ai eu la possibilité d'analyser ».

4 L'article est organisé de la façon suivante. La section 2 discute la délimitation des quatre classes morphosyntaxiques de lexèmes du soninké désignées ici comme « noms », « verbes ", « adjectifs » et " verbes statifs ». La section 3 décrit les différents types de constructions prédicatives du soninké selon la nature du terme en fonction prédicative. La section 4 discute la spécificité sémantique de l'emploi prédicatif de verbes statifs en contraste avec la prédication verbale et avec la prédication adjectivale. La section 5 propose un inventaire de verbes statifs. La section 6 discute le statut des verbes statifs dans le système de formation des mots du soninké. La section 7 récapitule les principales conclusions.

\section{Classes morphosyntaxiques de lexèmes}

\section{Noms, verbes, adjectifs et verbes statifs}

5 Comme on peut le constater d'après la liste de lexèmes simples classés par catégories qui figure dans (Creissels 2016), le soninké a deux types majeurs de lexèmes qui numériquement regroupent chacun un nombre de lexèmes très largement supérieur à celui des autres types de lexèmes que l'on peut reconnaître dans la langue : lexèmes nominaux et lexèmes verbaux. En outre, l'inventaire des lexèmes nominaux et des lexèmes verbaux peut être élargi à volonté au moyen d'opérations de morphologie constructionnelle (composition ou dérivation par affixation) qui pour la plupart ne sont pas limitées dans leur productivité.

6 À côté de ces deux catégories lexicales majeures, le soninké a la particularité de posséder deux classes de lexèmes qui regroupent toutes deux des lexèmes dont les signifiés sont du type qui caractérise les classes de lexèmes traditionnellement étiquetées «adjectifs » dans les grammaires descriptives. Comme nous le verrons plus loin, il y a un lien étroit entre ces deux classes au niveau des mécanismes de dérivation, mais en termes de caractéristiques tant morphologiques que syntaxiques, elles n'ont rien en commun. L'une présente des caractéristiques qui rappellent plutôt celles de la classe des verbes, tandis que l'autre a très nettement, de ces deux points de vue, des caractéristiques nominales.

7 Celle de ces deux catégories qui présente des caractéristiques morphosyntaxiques essentiellement nominales a toujours été étiquetée «adjectif» dans les travaux sur le soninké, et c'est ce terme que je continuerai d'utiliser ici. Pour celle qui présente des 
caractéristiques essentiellement verbales, j'utiliserai l'étiquette de "verbe statif », déjà utilisée pour une catégorie des langues mandingues aux caractéristiques très semblable à de nombreux égards.

8 Le choix de cette étiquette comporte toutefois un risque de malentendu, car elle peut suggérer que les verbes statifs seraient une sous-classe de la classe des verbes dont la délimitation reposerait sur un critère sémantique. Avant de poursuivre, j'insiste donc sur le fait que la distinction entre verbes et verbes statifs est établie sur la base de critères strictement morphosyntaxiques. Dans « verbe statif », «statif » ne doit pas être compris comme une caractérisation du signifié lexical des lexèmes regroupés sous cette étiquette, mais plutôt comme se référant au fait que, à la différence des verbes, les verbes statifs ont une flexion qui se limite à l'expression de la polarité (positif vs. négatif), à l'exclusion de toute distinction de type aspectuel ou modal.

\section{Les noms}

Morphologiquement, les noms du soninké sont typiquement caractérisés par un paradigme flexionnel qui comporte segmentalement cinq formes différentes :

- la forme non-autonome, utilisée exclusivement lorsque le lexème nominal fait partie d'un lexème construit dans lequel il n'occupe pas la position finale,

- le singulier non-déterminé,

- le singulier déterminé,

- le pluriel non-déterminé,

- le pluriel déterminé.

10 À ceci s'ajoutent deux formes tonales particulières :

- la forme du nom à ton grammatical BH est la 'forme construite' du nom, c'est-à-dire la forme utilisée si et seulement si le nom est la tête d'une construction génitivale ;

- le singulier non-déterminé à ton grammatical B s'utilise exclusivement pour la deuxième occurrence du lexème nominal dans la construction $\mathrm{N}$ wó $\mathrm{N}$ 'chaque $\mathrm{N}$, n'importe quel N', dans laquelle le lexème nominal doit être répété.

11 L'exemple suivant illustre avec yittè 'arbre' la totalité du paradigme flexionnel d'un nom soninké. Le $n$ entre parenthèses rend compte $d u$ fait que la marque de détermination nominale inclut une nasale qui toutefois n'est apparente que si le nom n'est pas immédiatement suivi de pause. ${ }^{2}$

Tableau 1 : Paradigme flexionnel de yíttè 'arbre'

\begin{tabular}{|l|l|l|l|}
\hline & forme non-autonome & & yittí- \\
\hline & singulier non dét. & & yitté \\
\hline & singulier non dét. + ton gramm. B & & yittè \\
\hline & singulier non dét. + ton gramm. BH & & yitté \\
\hline singulier dét. & & yittè $(n)$ \\
\hline & singulier dét. + ton gramm. BH & & yittê( $(n)$ \\
\hline
\end{tabular}




\begin{tabular}{|c|c|}
\hline pluriel non dét. & yittú \\
\hline pluriel non dét. + ton gramm. BH & yittú \\
\hline pluriel dét. & yittù(n) \\
\hline pluriel dét. + ton gramm. $\mathrm{BH}$ & yittû(n) \\
\hline
\end{tabular}

Les noms du soninké se répartissent en un certain nombre de classes flexionnelles. Ousmane Diagana (1995) en dénombre 21 pour le soninké de Kaédi. Certaines sont très productives, d'autres ne comportent qu'un tout petit nombre de noms, et sont susceptibles de disparaître par réaffectation des quelques noms qu'elles comportent à des classes plus productives. Toutefois la donnée de trois formes seulement suffit à prédire la totalité du paradigme : la forme non-autonome, le singulier non-déterminé et le pluriel non-déterminé. À partir de là on peut prédire automatiquement les formes déterminées ainsi que les formes tonalement modifiées par un ton grammatical. Par exemple, la classe flexionnelle à laquelle appartient yitte 'arbre' se définit par l'alternance entre $i$ (forme non-autonome), $e$ (singulier) et $u$ (pluriel). En ce qui concerne le schème tonal, on peut toujours prédire le ton de la forme non-autonome à partir de celui de la forme du singulier, mais pas l'inverse. ${ }^{3}$ Quant au ton de la forme de pluriel, si le pluriel est formé par alternance vocalique, son ton est identique à celui de la forme de singulier ; si le pluriel est formé par suffixation, le suffixe de pluriel -nú ou -ní s'ajoute à la forme de singulier dont le ton ne subit aucune modification.

Syntaxiquement, les noms fonctionnent comme noyaux de groupes nominaux dont l'accès aux fonctions de sujet, objet, oblique et génitif est seulement limité par des questions de cohérence sémantique. Les lexèmes nominaux n'ont par contre pas accès à la position $\mathrm{V}$ dans la construction prédicative verbale $\mathrm{S} \mathrm{mp}(\mathrm{O}) \mathrm{V}(\mathrm{X}){ }^{4} \mathrm{Il}$ y a bien quelques rares noms dont la forme coïncide avec celle d'un lexème verbal apparenté, mais le caractère à la fois exceptionnel et imprédictible de cette situation suggère de voir là un phénomène de conversion lexicalement déterminé.

\section{Les verbes}

14 Morphologiquement, la flexion verbale du soninké se limite aux deux distinctions suivantes, qui se croisent pour donner un paradigme de quatre formes fléchies :

- la distinction entre le lexème verbal nu et une forme suffixée désignée ici comme " gérondif ",

- l'alternance entre le schème tonal lexical du verbe et le schème tonal entièrement $B$.

La flexion verbale est illustrée ci-dessous avec trois verbes représentant les trois classes tonales de verbes dissyllabiques que connaît le soninké5:

Tableau 2 : Paradigme flexionnel de qóbó 'acheter', gáagà 'vendre' et siití 'attacher'

\begin{tabular}{|l|l|l|l|l|l|l|}
\hline & 'acheter' & 'vendre’ & 'attacher' & \\
\hline lexème nu & qóbó & gáagà & siití & \\
\hline
\end{tabular}




\begin{tabular}{|l|l|l|l|l|l|l|}
\hline lexème nu + ton gramm. B & qòbò & gàagà & sìitì & \\
\hline gérondif & qóbó-nó & gáagà-ná & sìitì-ní & \\
\hline gérondif + ton gramm. B & qòbò-nò & gàagàà̀a & sìitì-nì & \\
\hline
\end{tabular}

16 L'exemple (1) illustre la flexion tonale du verbe dans les phrases dont un terme est focalisé. Dans ce contexte, le verbe a son schème tonal inhérent s'il précède le terme focalisé, comme en (c), alors qu'il a le ton grammatical B s'il lui succède, comme en (a) ou (b).

\begin{tabular}{|l|l|l|l|l|l|l|l|}
\hline (1a) & Múusá & yà-n & dà & qálisí-n & kinì & Hàatú & yì. \\
\hline & Moussa & FOC-SUJ & TR & argent-D & donner & Fatou & POSTP \\
\hline
\end{tabular}

\begin{tabular}{|l|l|l|l|l|l|l|l|}
\hline (1b) & Múusá & dà & qúlisí-n & $\tilde{n} a ̀$ & kinì & Hàatú & yì. \\
\hline & Moussa & TR & argent-D & FOC & donner & Fatou & POSTP \\
\hline \multicolumn{7}{|l}{} \\
'C'est l'argent que Moussa a donné à Fatou.' \\
\hline
\end{tabular}

\begin{tabular}{|l|l|l|l|l|l|l|l|}
\hline (1c) & Múusá & dà & qálisí-n & kíìi & Hàatú & yà & yí. \\
\hline & Moussa & TR & argent-D & donner & Fatou & FOC & POSTP \\
\hline
\end{tabular}

17 Les lexèmes verbaux ont accès à la position $\mathrm{V}$ dans la construction prédicative verbale $\mathrm{S} m p(0) \mathrm{V}(\mathrm{X})$. Dans cette construction, il y a une relation particulière de conditionnement mutuel entre les positions $\mathrm{mp}$ et $\mathrm{V}$ : à l'exception de ntá, chaque marqueur prédicatif est compatible avec l'une seule des quatre formes fléchies du verbe (et réciproquement, chaque forme fléchie du verbe est compatible avec certains marqueurs prédicatifs seulement - parfois un seul).

\section{Les adjectifs}

Les adjectifs ne se distinguent pas des noms au niveau de leur flexion. Ils peuvent aussi occuper à eux seuls toutes les positions syntaxiques typiquement occupées par des noms, mais avec une restriction importante: exactement comme les adjectifs du français et des autres langues romanes, un adjectif soninké ne peut occuper à lui seul une position nominale (notamment la position de terme sujet, objet ou oblique d'une construction prédicative) que si le contexte permet de l'interpréter comme modifiant sémantiquement un lexème nominal non exprimé. Une phrase comme (2), où la position d'objet est occupée par un adjectif, ne peut s'utiliser que dans un contexte 
permettant de deviner le type d'entité auquel s'applique la qualification 'blanc / blanche' : tissu, cheval, vache, etc.

\begin{tabular}{|l|l|l|l|l|l|l|}
\hline$(2)$ & A & dà & qúllè-n & qóbó. & & \\
\hline & $3 \mathrm{SG}$ & TR & blanc & acheter & & \\
\hline \multicolumn{7}{|l|}{} \\
\multicolumn{7}{|l|}{ 'Il a acheté le blanc / la blanche.' } \\
\hline
\end{tabular}

19 Par contre, lorsqu'ils se combinent avec des noms qu'ils qualifient, les adjectifs entrent dans une construction particulière qui fait leur spécificité par rapport aux noms. En effet, ils forment avec les noms qu'ils qualifient des composés morphologiques dont la structure est différente de celle des composés dans lesquels un nom modifie un autre nom - exemple (3).

\begin{tabular}{|l|l|l|l|l|}
\hline$(3)$ & A & dà & sélin-qúlle-n & qóbó. \\
\hline & $3 S G$ & TR & poule-blanc-D & acheter \\
\hline & \multicolumn{4}{|c|}{ 'Il a acheté la poule blanche.' } \\
\hline
\end{tabular}

À l'exemple (3), sélinyê est à la même forme non-autonome sélin- que dans des composés $\mathrm{N}+\mathrm{N}$ tels que sélin-kánpè 'aile de poule' ou sélin-gáagàanâ 'vendeur de poules', mais les composés dans lesquels un nom modifie un autre nom sont construits selon l'ordre 'modifieur suivi du modifié' (qui est aussi celui de la construction génitivale ou possession adnominale), tandis que les composés dans lesquels un adjectif modifie un nom sont construits selon l'ordre 'modifié suivi du modifieur'.

En outre, dans leur emploi prédicatif, les adjectifs ne se comportent pas exactement comme les noms: comme nous le verrons en 3.6, c'est la même copule équative qui marque l'emploi prédicatif des noms et des adjectifs, mais la construction est différente.

Par contraste avec les langues mandingues, dans lesquelles une partie seulement des lexèmes correspondants forment des composés avec les noms qu'ils qualifient, il est important de souligner qu'en soninké, quelle que soit la nature morphologique précise des adjectifs (simples, dérivés par affixation ou composés), ils ne peuvent fonctionner comme modifieurs qualificatifs de noms que dans le cadre de composés dans lesquels le nom qualifié est à la forme non-autonome, et auxquels s'applique la règle de compacité tonale qui vaut pour tous les autres types de composés nominaux. Comme on peut le voir à l'exemple (4), en soninké, lorsqu'un nom et un adjectif se succèdent sans former un composé, il ne peut pas s'agir d'une construction dans laquelle l'adjectif restreindrait le signifié lexical du nom qui le précède, mais uniquement d'une construction dans laquelle l'adjectif est en fonction de prédicat second, le nom auquel il succède étant nécessairement à la forme définie.

\begin{tabular}{|l|l|l|l|l|l|l|l|l|l|l|}
\hline$(4 \mathrm{a})$ & $\hat{N}$ & dà & yàxàrì-ñáxálintè-n & yàrí. & & & & & & \\
\hline
\end{tabular}




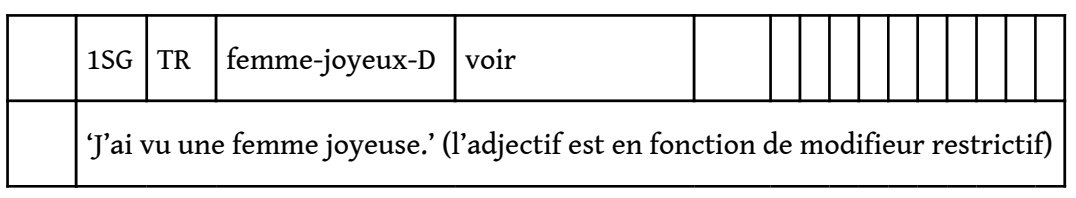

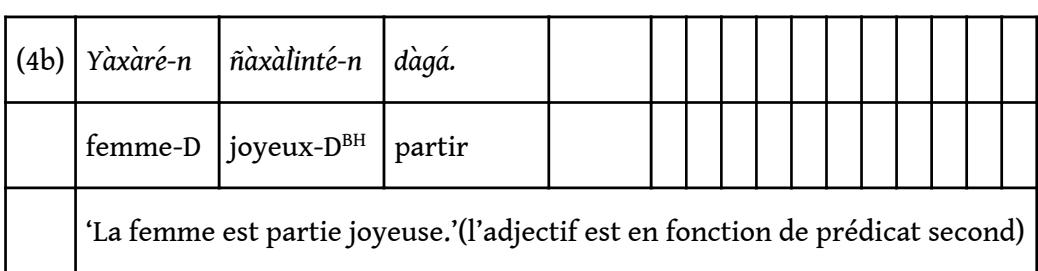

\section{Les verbes statifs}

Les verbes statifs fonctionnent prédicativement dans une construction qui n'est pas différente de la construction prédicative verbale intransitive $\mathrm{S} \mathrm{mp} \mathrm{V}(\mathrm{X})$, avec toutefois les deux particularités suivantes qui les distinguent des verbes:

- les verbes statifs n'ont pas de forme de gérondif,

- comme nous le verrons plus en détail en 3.3, le paradigme des marqueurs prédicatifs compatibles avec les verbes statifs se réduit à deux éléments dont l'opposition se limite à exprimer la distinction "positif vs. négatif ", à l'exclusion des valeurs aspectuelles ou modales que ces mêmes marqueurs prédicatifs expriment dans leur combinaison avec les verbes.

\section{Constructions prédicatives}

\section{La construction prédicative verbale}

La construction prédicative verbale peut être schématisée par la formule suivante, où $\mathrm{S}$, $\mathrm{mp}, \mathrm{O}$, V et $\mathrm{X}^{*}$ sont mis respectivement pour « constituant nominal sujet ", " marqueur prédicatif», «constituant nominal objet», «verbe», et «un ou plusieurs termes obliques ».

$\mathrm{S} \mathrm{mp}(\mathrm{O}) \mathrm{V}\left(\mathrm{X}^{*}\right)$

Selon que le terme $O$ est ou non présent, on peut parler de construction prédicative verbale transitive ou intransitive. Le soninké fait partie des langues dans lesquelles la distinction entre construction transitive et construction intransitive est formellement marquée. En effet, plusieurs valeurs de TAM s'expriment différemment selon que la construction est transitive ou intransitive :

- à l'accompli positif, la position du marqueur prédicatif reste vide si la construction est intransitive, alors qu'elle est occupée par le marqueur de transitivité dà si la construction est transitive - ex. (5) ;

-à l'impératif positif, si le sujet est singulier, la position du marqueur prédicatif reste dans tous les cas vide; par contre si le sujet est pluriel, elle n'est vide qu'à l'intransitif, tandis qu'au transitif elle est occupée par le même marqueur de transitivité dà - ex. (6) ;

- le marqueur prédicatif du subjonctif est nàn ou ' $n$ (en variation libre) si la construction est intransitive, nà si la construction est transitive - ex. (7); 
- à l'inaccompli positif, en présence d'un constituant focalisé ou d'un mot interrogatif, la position du marqueur prédicatif reste vide si la construction est intransitive, alors qu'elle est occupée par nà si la construction est transitive - ex. (8).

\begin{tabular}{|l|l|l|l|l|}
\hline (5a) & Hàré-n & dùgùtá. & & \\
\hline & âne-D & s'échapper & & \\
\hline & 'L'âne s'est échappé.' \\
\hline
\end{tabular}

\begin{tabular}{|l|l|l|l|l|}
\hline (5b) & Hàré-n & dà & yúgò-n & dànbú. \\
\hline & âne-D & TR & homme-D & donner_un_coup_de_pied \\
\hline & \multicolumn{3}{|l}{ 'L'âne a donné un coup de patte à l'homme.' } \\
\hline
\end{tabular}

\begin{tabular}{|l|l|l|l|l|}
\hline (6a) & Qà & táaxú ! & & \\
\hline & $2 \mathrm{PL}^{\mathrm{B}}$ & s'asseoir & & \\
\hline \multicolumn{3}{|l|}{ 'Asseyez-vous !' } \\
\hline
\end{tabular}

\begin{tabular}{|l|l|l|l|l|l|}
\hline$(6 \mathrm{~b})$ & Qà & dà & yillé & ké & ró-ndí ! \\
\hline & $2 \mathrm{PL}^{\mathrm{B}}$ & $\mathrm{TR}$ & mil & $\mathrm{DEM}$ & entrer-CAUS \\
\hline \multicolumn{6}{|l}{ 'Faites rentrer le mil !' } \\
\hline
\end{tabular}

\begin{tabular}{|l|l|l|l|l|l|}
\hline$(7 \mathrm{a})$ & I & tí & án & nàn & táaxú. \\
\hline & 3PL & dire & 2SG & SUBJ.INTR & s'asseoir \\
\hline \multicolumn{6}{|l}{} \\
\hline
\end{tabular}

\begin{tabular}{|l|l|l|l|l|l|l|}
\hline$(7 \mathrm{~b})$ & I & tí & án & nà & Hàatú & deemá. \\
\hline & 3PL & dire & $2 \mathrm{SG}$ & SUBJ.TR & Fatou & aider \\
\hline \multicolumn{7}{|l}{ 'On te dit d'aider Fatou.' } \\
\hline
\end{tabular}

\begin{tabular}{|c|c|c|c|}
\hline (8a) & A & wá & sálti-ní. \\
\hline & $3 S G$ & INACP & prier-GER \\
\hline & \multicolumn{3}{|c|}{ 'Il prie.' } \\
\hline
\end{tabular}




\begin{tabular}{|l|l|l|l|l|l|}
\hline$(8 \mathrm{~b})$ & A & $\varnothing$ & sálli-ní & yà. & \\
\hline & 3SG & INACP.INTR. & prier-GER & FOC & \\
\hline \multicolumn{4}{|c}{} \\
\multicolumn{3}{|l}{ 'Ce qu'il fait, c'est prier.' } \\
\hline
\end{tabular}

\begin{tabular}{|l|l|l|l|l|l|}
\hline$(8 \mathrm{c})$ & A & wá & hàrê-n & gáagà-ná. & \\
\hline & 3SG & INACP & âne-D & vendre-GER & \\
\hline & \multicolumn{2}{|l}{ 'Il vend l'âne.' } \\
\hline
\end{tabular}

\begin{tabular}{|l|l|l|l|l|l|}
\hline$(8 \mathrm{~d})$ & A & nà & hàrê-n & gáagà-ná & yà. \\
\hline & $3 S \mathrm{SG}$ & INACP.TR & âne-D & vendre-GER & FOC \\
\hline & \multicolumn{7}{|c}{ 'Ce qu'il fait avec l'âne, c'est de le vendre.' } \\
\hline
\end{tabular}

\section{Marqueurs prédicatifs combinables aux verbes}

On se limite ici aux marqueurs prédicatifs susceptibles de figurer dans une phrase indépendante. Les marqueurs prédicatifs présentés dans le Tableau 3 se combinent à la forme nue du verbe.

Les marqueurs prédicatifs présentés dans le Tableau 4 se combinent à la forme de gérondif du verbe.

Tableau 3 : Les marqueurs prédicatifs verbaux combinables à la forme nue du verbe

\begin{tabular}{|l|l|l|l|l|}
\hline accompli positif, intransitif & & & $\emptyset$ \\
\hline accompli positif, transitif & & & dà & \\
\hline accompli négatif & & & má & \\
\hline accompli récent & & & háxà & \\
\hline accompli passé & & & 'ñigà & \\
\hline accompli passé négatif & & má n̂̀igà & \\
\hline & & & & \\
\hline projectif positif & ná & \\
\hline projectif négatif & & & ntá & \\
\hline & & & & \\
\hline subjonctif positif, intransitif & & & & \\
\hline
\end{tabular}




\begin{tabular}{|c|c|}
\hline subjonctif positif, transitif & $n \grave{a}$ \\
\hline subjonctif négatif & nàn máxà \\
\hline impératif positif, intransitif & $\varnothing$ \\
\hline impératif positif, transitif ${ }^{8}$ & $\varnothing /$ dà \\
\hline impératif négatif & máxà \\
\hline
\end{tabular}

Tableau 4 : Les marqueurs prédicatifs verbaux combinables à la forme de gérondif

\begin{tabular}{|l|l|l|l|l|}
\hline inaccompli positif $1^{9}$ & & wá & \\
\hline & inaccompli positif 2, intransitif & & $\varnothing$ & \\
\hline & inaccompli positif 2, transitif & & nà & \\
\hline inaccompli négatif & & ntá & \\
\hline inaccompli passé & & 'ñi & \\
\hline inaccompli passé négatif & má ñi & \\
\hline progressif ${ }^{10}$ & & háýi & \\
\hline
\end{tabular}

Les marqueurs prédicatifs má et ntá ont la particularité d'imposer au verbe la forme à ton grammatical B, alors qu'avec tous les autres marqueurs prédicatifs susceptibles de figurer dans une phrase indépendante, l'emploi de la forme à ton grammatical B est conditionné par la présence d'un constituant nominal focalisé ou d'un mot interrogatif en fonction de sujet ou d'objet.

\section{Marqueurs prédicatifs combinables aux verbes statifs}

Les lexèmes appartenant à la classe des verbes statifs occupent la position $\mathrm{V}$ dans une construction prédicative dont la structure est celle de la prédication verbale intransitive, mais ils se combinent avec un couple unique de marqueurs prédicatifs (nàn ̀n 'positif', glosé 'ST' et ntá 'négatif', glosé 'ST.NEG'), et leur flexion se réduit à la flexion tonale «forme à ton lexical» (si le marqueur prédicatif est nàn ' $n$ et que le sujet n'est pas focalisé) vs. "forme à ton grammatical B » (si le marqueur prédicatif est ntá, ou si le sujet est focalisé). Les exemples (9) et (10) illustrent quelques verbes statifs.

\begin{tabular}{|c|c|c|c|c|c|c|}
\hline (9a) & Ń & qàràllènmú- $n$ & nàn & púnyù & tánñéré & yì. \\
\hline & $1 S G$ & élève.PL-D ${ }^{\mathrm{BH}}$ & ST & être_supérieur & soixante-dix & POSTP \\
\hline
\end{tabular}


'Mes élèves sont plus de soixante-dix.'

\begin{tabular}{|l|l|l|l|l|l|}
\hline (9b) & Dèbì-gòori-yé-n & nàn & pàsú & jàatììi-gòorì-yé-n & jà. \\
\hline & village-éviter-NMLS-D & ST & être_meilleur & logeur-éviter-NMLS-D & POSTP \\
\hline & 'Il vaut mieux éviter un village qu'éviter son logeur.' \\
\hline
\end{tabular}

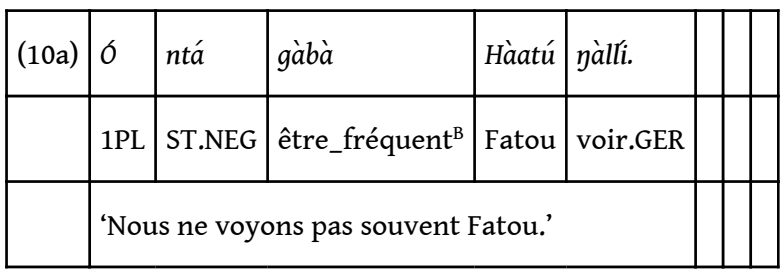

\begin{tabular}{|l|l|l|l|l|l|}
\hline$(10 b)$ & Ñengèntàaxû- $n$ & $n t a ́$ & linji & Allà & dá. \\
\hline & injustice-D & ST.NEG & être_agréable $^{\mathrm{B}}$ & Dieu & pour \\
\hline & 'Dieu n'aime pas l'injustice.' \\
\hline
\end{tabular}

31 Les deux marqueurs prédicatifs combinables avec les verbes statifs (nàn ̀̀ 'positif' et ntá 'négatif') existent aussi en combinaison avec les verbes, mais appariés différemment, et avec d'autres valeurs sémantiques :

- avec les verbes, le prédicatif positif nàn ǹ a une valeur de subjonctif, et forme un couple avec le prédicatif négatif nàn máxà (et non pas ntá);

- avec les verbes, le prédicatif négatif ntá combiné à la forme nue du lexème verbal a une valeur de projectif, et forme un couple avec le prédicatif positif ná (et non pas nàn $\sim \grave{n}$ ).

Le point crucial est que, combinés aux verbes statifs, nàn $\sim \grave{n}$ et ntá se limitent à marquer la distinction de polarité (positif vs. négatif), et sont totalement dépourvus des valeurs aspecto-modales qu'ils expriment avec les verbes.

\section{La prédication non verbale de localisation}

La copule de localisation wá (positif) / ntá (négatif) doit minimalement être combinée à deux termes. L'un précède la copule, et dans l'emploi prototypique de cette construction, il représente une entité qu'on localise. L'autre suit la copule, et il ne se distingue en rien des obliques qui succèdent au verbe dans la prédication verbale; dans l'emploi prototypique de cette construction, il exprime la localisation du référent de l'autre terme.

\begin{tabular}{|l|l|l|l|l|l|}
\hline (11a) & Hàatú & wá & kónpè-n & di. & \\
\hline & Fatou & COPLOC & chambre-D & dans & \\
\hline & \multicolumn{3}{|l}{ 'Fatou est dans la chambre.' } \\
\hline
\end{tabular}




\begin{tabular}{|l|l|l|l|l|l|}
\hline (11b) & Hàatú & ntá & kónpè-n & dí. & \\
\hline & Fatou & COPLOC.NEG & chambre-D & dans & \\
\hline & \multicolumn{3}{|l}{ 'Fatou n'est pas dans la chambre.' } \\
\hline
\end{tabular}

La copule de localisation positive a une variante nà dans les phrases comportant un groupe nominal focalisé ou un mot interrogatif.

\begin{tabular}{|l|l|l|l|l|l|}
\hline (12a) & Kó & nà & kónpè-n & dì ? & \\
\hline & qui & COPLOC & chambre-D & dans & \\
\hline \multicolumn{4}{|c|}{ 'Qui est dans la chambre?’ } \\
\hline
\end{tabular}

\begin{tabular}{|l|l|l|l|l|l|}
\hline (12b) & Hàatú & yà & nà & kónpè-n & di. \\
\hline & Fatou & FOC & COPLOC & chambre-D & dans \\
\hline & 'C'est Fatou qui est dans la chambre.' \\
\hline
\end{tabular}

\begin{tabular}{|l|l|l|l|l|}
\hline (12c) & Hàatú & nà & mínnà ? & \\
\hline & qui & COPLOC & où & \\
\hline \multicolumn{3}{|l|}{ 'Où est Fatou?' } \\
\hline
\end{tabular}

\begin{tabular}{|l|l|l|l|l|l|l|}
\hline (12d) & Hàatú & nà & ké & kónpé & yà & dì. \\
\hline & Fatou & COPLOC & DEM & chambre & FOC & dans \\
\hline & 'C'est dans cette chambre qu'est Fatou.' \\
\hline
\end{tabular}

Il y a une relation évidente entre la copule de localisation et le marqueur prédicatif d'inaccompli dans la construction prédicative verbale, qui doivent toutefois être distingués dans une analyse synchronique. En effet :

- ntá en tant que marqueur prédicatif d'inaccompli modifie la tonalité du verbe de la même façon que le marqueur prédicatif d'accompli négatif má, alors que dans son emploi copulatif, ntá n'exerce aucune influence tonale sur les autres mots présents dans la construction;

-à l'intransitif en présence d'un groupe nominal focalisé ou d'un mot interrogatif, le marqueur d'inaccompli wá a une variante zéro, alors qu'il n'y a rien de tel pour la copule de localisation. 


\section{La prédication non verbale d'identification}

La copule équative 'ní (positif) / 'hé hèti (négatif) figure dans une construction qui admet deux variantes selon que l'entité identifiée ou catégorisée est explicitement désignée ou reste sous-entendue.

Si l'entité que l'on identifie n'est pas explicitement désignée, le constituant exprimant l'identification ou la catégorisation précède la copule équative, et dans une phrase positive indépendante, il doit être marqué du focalisateur 'yá.

\begin{tabular}{|c|c|c|c|}
\hline (13) & Tàgé-n & $\tilde{n} \grave{a}$ & ní. \\
\hline & forgeron-D & FOC & COPEQ \\
\hline & \multicolumn{3}{|c|}{ 'C'est un forgeron.' } \\
\hline
\end{tabular}

Si l'entité que l'on identifie est explicitement désignée, la copule d'identification doit être précédée d'un constituant nominal et suivie d'un autre constituant nominal marqué de la postposition 'yí 'yá 'yá, mais la désignation de l'entité identifiée et l'identification n'occupent pas une position fixe dans cette construction. Dans les phrases positives indépendantes, l'un des deux termes doit être marqué du focalisateur 'yá, et c'est la place du focalisateur qui est déterminante pour l'interprétation - ex. (14).

\begin{tabular}{|l|l|l|l|l|l|l|}
\hline$(14 \mathrm{a})$ & Ké & yúgó & nì & tàgé-n & $\tilde{n} a ̀$ & yí. \\
\hline & DEM & homme & COPEQ & forgeron-D & FOC & POSTP \\
\hline \multicolumn{7}{|l}{ 'Cet homme est forgeron.' } \\
\hline
\end{tabular}

\begin{tabular}{|l|l|l|l|l|l|l|}
\hline$(14 \mathrm{~b})$ & Tàgé-n & $\tilde{n} a ̀$ & $n i ́$ & ké & yúgó & yì. \\
\hline & forgeron-D & FOC & COPEQ & DEM & homme & POSTP \\
\hline & 'Cet homme est forgeron.' \\
\hline
\end{tabular}

\section{Adjectifs en fonction prédicative}

Les adjectifs ont en commun avec les noms d'assumer la fonction prédicative en se combinant à la copule équative. Toutefois, la construction n'est pas identique à celle décrite en 3.5 pour les noms. En effet, dans la prédication adjectivale, la copule équative est obligatoirement précédée de deux termes, et la présence du focalisateur ne constitue pas une obligation.

Le premier terme de la construction prédicative propre aux adjectifs est un constituant nominal dont on prédique la propriété signifiée par l'adjectif, et le deuxième est l'adjectif en fonction de prédicat. Curieusement, l'adjectif en fonction de prédicat et le 
constituant nominal qui le précède sont mis en forme exactement comme le nom-tête et son modifieur dans une construction génitivale..$^{11} \mathrm{En}$ effet, quel que soit son schème tonal inhérent, l'adjectif en fonction prédicative prend uniformément le schème tonal BH qui est aussi celui de la forme construite des noms, et s'il est précédé d'un pronom de troisième personne, celui-ci prend la forme à ton $\mathrm{H}$, exactement comme en fonction de possesseur adnominal. Par exemple, l'adjectif illustré en emploi prédicatif à l'exemple (15) a pour schème tonal lexical tóoxintè.

\begin{tabular}{|l|l|l|l|l|}
\hline (15a) & Nántò-n & bùnné-n & tòoxinté-n & nì. \\
\hline & scorpion-D & piqûre- $\mathrm{D}^{\mathrm{BH}}$ & douloureux- $\mathrm{D}^{\mathrm{BH}}$ & COPEQ \\
\hline & \multicolumn{3}{|l}{ 'La piqûre du scorpion est douloureuse.' } \\
\hline
\end{tabular}

\begin{tabular}{|l|l|l|l|l|}
\hline$(15 b)$ & $A$ & tooxinté- $n$ & ni. & \\
\hline & $3 S G^{\mathrm{H}}$ & douloureux- $\mathrm{D}^{\mathrm{BH}}$ & COPEQ & \\
\hline & 'C'est douloureux.' \\
\hline
\end{tabular}

\section{Implications sémantiques de l'emploi de verbes statifs}

Comme cela apparaîtra dans l'inventaire de verbes statifs fourni à la section 5, en soninké, il n'y a que très peu de verbes statifs qui ne soient pas en concurrence avec un adjectif et un verbe auxquels ils sont apparentés. En outre en soninké, comme cela est courant dans les langues d'Afrique de l'Ouest, le paradigme flexionnel des verbes signifiant des processus comporte des formes couramment étiquetées « accompli » (ou «perfectif ») largement disponibles pour se référer non seulement à l'aboutissement de processus, mais aussi à des états abstraction faite de toute implication quant à un processus dont ces états pourraient résulter. Ainsi, à l'exemple (16), l'accompli négatif de qótò 'devenir difficile' ne doit pas être compris comme 'n'est pas devenu difficile', mais plutôt comme 'n'est pas difficile', et on pourrait multiplier à l'infini de tels exemples.

\begin{tabular}{|l|l|l|l|l|}
\hline$(16)$ & Nàn & ñigé & má & qòtò. \\
\hline & INF.INTR & manger.ANTIP & ACP.NEG & devenir_difficile \\
\hline & \multicolumn{3}{|l}{ 'Ce n'est pas difficile de manger.' } \\
\hline
\end{tabular}

Dans ces conditions, on doit se demander ce qui peut conditionner l'utilisation de verbes statifs de préférence à l'accompli d'un verbe de processus, ou à une prédication adjectivale. O. M. Diagana (1995:263) évoque à juste titre les notions d'intensité et d'emphase. On pourrait ajouter la notion d'évaluation. En effet, il est tout à fait évident que dans les séances d'élicitation, les locuteurs sont particulièrement enclins à 
produire des verbes statifs lorsqu'on leur demande d'exprimer des comparaisons, comme à l'exemple (17).

\begin{tabular}{|c|c|c|c|c|c|c|c|}
\hline (17) & Kànjàané-n & tàrú-n & nàn & giilá & gùwàané-n & qàllú-n & yà. \\
\hline & lièvre-D & oreille.PL-D ${ }^{\mathrm{BH}}$ & ST & être_long & écureuil-D & part.PL-D ${ }^{\mathrm{BH}}$ & POSTP \\
\hline
\end{tabular}

\section{L'inventaire des verbes statifs}

semble le suggérer O. M. Diagana (1995:264), il n'est pas facile d'établir de façon précise l'inventaire des lexèmes du soninké susceptibles d'avoir le comportement typique des verbes statifs. En effet, comme cela apparaitra dans l'inventaire ci-dessous, l'immense majorité des verbes statifs du soninké sont apparentés à la fois à un verbe et à un adjectif dont ils diffèrent essentiellement par leur voyelle finale, et cette situation favorise indéniablement la création accidentelle de verbes statifs en plus de ceux dont l'usage est consacré. Ainsi, en situation d'élicitation, les consultants peuvent avoir du mal à évaluer s'il existe ou non un verbe statif correspondant à tel verbe ou adjectif, et revenir d'une séance à l'autre sur leurs jugements. C'est pour cette raison que, dans l'inventaire qui suit, j'ai choisi de ne pas donner la liste de tous les verbes statifs que j'ai pu obtenir en élicitation, et de me limiter à ceux qui sont aussi attestés par au moins une autre source dans une phrase où on ne peut les analyser que comme des verbes statifs. ${ }^{12}$ Par souci d'homogénéité des données, je ne tiens pas compte non plus de quelques verbes statifs que j'ai notés dans diverses publications mais que je n'ai pas obtenus dans ma propre enquête. Cet inventaire est donc certainement incomplet, et en outre il ne préjuge en rien de la possibilité de variations dans l'inventaire des verbes statifs d'un parler à un autre.

\section{Verbes statifs « primaires »}

Les verbes statifs énumérés à cette section se distinguent de ceux décrits en 5.2 par le fait qu'ils ne sont pas de manière évidente le produit d'une dérivation par affixation. Si «primaire » est toutefois mis entre guillemets, c'est parce que, comme cela a déjà été dit, la plupart des verbes statifs « primaires » entretiennent une relation avec un verbe et avec un adjectif, mais une relation qui n'a pas d'orientation évidente, car elle met essentiellement en jeu des alternances de voyelles.

\begin{tabular}{|l|l|l|l|l|l|l|l|l|l|}
\hline$(18)$ & A & dò & sèré & tàná & ntá & bàanà & în & màxá. \\
\hline & & $3 S G^{\mathrm{H}}$ & avec & personne & autre & ST.NEG & être_égal $^{\mathrm{B}}$ & $1 \mathrm{SG}$ & pour \\
\hline \multicolumn{6}{|l}{} \\
\hline
\end{tabular}


cf. báané 'un', báanò 'devenir identique'

- báráxátá 'être gros, fier'

\begin{tabular}{|l|l|l|l|l|l|l|l|l|l|}
\hline & $(19)$ & Múusá & yàn & nàn & bàràxàtà & Sánbà & yí. & & \\
\hline & Moussa & FOC & ST & être_gros $^{\mathrm{B}}$ & Samba & POSTP & & \\
\hline \multicolumn{6}{|c|}{} \\
\hline
\end{tabular}

cf. báráxàtê 'gros', báráxátó 'grossir'

- dàrá 'être gros, important'

\begin{tabular}{|l|l|l|l|l|l|l|l|}
\hline & $(20)$ & Kiidé-n & nàn & dàrá, & qà & á & gingê-n \\
\hline & & baobab-D & ST & être_gros & mais & $3 \mathrm{SG}^{\mathrm{H}}$ & branche- $\mathrm{D}^{\mathrm{BH}}$ \\
\hline
\end{tabular}

\begin{tabular}{|l|l|l|l|l|l|}
\hline & & ntá & ñàa-nà & làbù-n-qáré & ỳ̀. \\
\hline & & INACP.NEG & faire-GER & couteau-EP-manche & POST \\
\hline & & 'Le baobab est énorme, mais sa branche ne peut pas s'utiliser comme manche de couteau.' \\
\hline
\end{tabular}

\section{cf. dàrê 'important', dàré 'devenir important', dàró 'accorder de l'importance à'}

- dehá 'être court, bas'

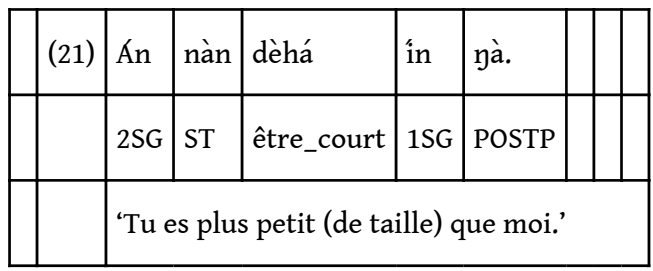

\section{cf. deppê 'court, bas', dehó ‘devenir court, bas'}

\section{- dinká 'être gros'}

\begin{tabular}{|l|l|l|l|l|l|l|l|l|}
\hline & (22) & Án & nàn & dinká & ín & yà. & & \\
\hline & & $2 S G$ & ST & être_gros & 1SG & POSTP & & \\
\hline & \multicolumn{6}{|c|}{ 'Tu es plus gros que moi.' } \\
\hline
\end{tabular}

\section{cf. dinkâ 'gros', dinkó 'grossir'}

- gàbá 'être souvent quelque part, faire souvent quelque chose' 


\begin{tabular}{|l|l|l|l|l|l|l|l|l|l|}
\hline (23) & À & nàn & gàbá & ké & yítté & yà & wùré. & \\
\hline & & $3 S G$ & ST & être_fréquent & DEM & arbre & FOC & sous & \\
\hline & \multicolumn{6}{|c|}{} \\
\hline
\end{tabular}

\begin{tabular}{|l|l|l|l|l|l|l|l|l|l|}
\hline & (26) & Ádámá & nàn & púnyù & Múusá & ỳi & qálisí & yà. & \\
\hline & & Adama & ST & être_supérieur & Moussa & POSTP & argent & POSTP & \\
\hline \multicolumn{6}{|l|}{ 'Adama a plus d'argent que Moussa.' } \\
\hline
\end{tabular}

\begin{tabular}{|l|l|l|l|l|l|l|l|l|}
\hline & $(27)$ & Sí̀-n & nàn & jàwú & hàré-n & yà. & & \\
\hline & & cheval-D & ST & être_rapide & âne-D & POSTP & & \\
\hline & & \multicolumn{7}{|l|}{ 'Le cheval est plus rapide que l'âne.' } \\
\hline
\end{tabular}

cf. jayê 'rapide', jàwó 'devenir rapide' - giilá 'être long, haut'

\begin{tabular}{|l|l|l|l|l|l|l|l|l|}
\hline & $(24)$ & Múusá & nàn & giilá & Dénbà & yí. & & \\
\hline & & Moussa & ST & être_long & Demba & POSTP & & \\
\hline & \multicolumn{6}{|c|}{ 'Moussa est plus grand (de taille) que Demba.' } \\
\hline
\end{tabular}

cf. giilêe 'long, haut', giiiló 'devenir long, haut'

• hàsú 'être meilleur, aller mieux'

\begin{tabular}{|l|l|l|l|l|l|l|l|l|}
\hline (25) & Mènjàn-círè-n & nàn & pàsú & wáaxí-búrè-n & yá. & & \\
\hline & ami-bon-D & ST & être_meilleur & frère-mauvais-D & POSTP & & \\
\hline & & 'Un bon ami vaut mieux qu'un mauvais frère.' & \\
\hline
\end{tabular}

cf. hàsê 'meilleur', hàsó 's'améliorer'

• húnyù 'être supérieur'

\section{cf. húnฑò ‘dépasser’13}

- jàwú 'être rapide'

cf. gàbê 'nombreux, abondant, fréquent', gàbó 'devenir nombreux, abondant, fréquent' 
- láatú 'être loin'

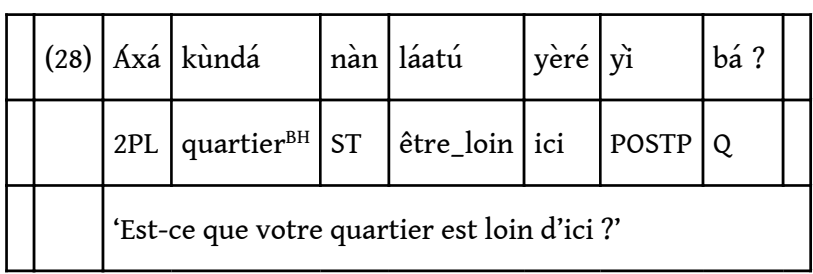

\begin{tabular}{|l|l|l|l|l|l|l|l|l|l|}
\hline (32) & Ké & góllé & nàn & nèhá & ké & báanè & yí. & \\
\hline & & DEM & travail & ST & être_facile & DEM & un & POSTP & \\
\hline \multicolumn{7}{|c|}{ 'Ce travail est plus facile que cet autre.' }
\end{tabular}




\begin{tabular}{|l|l|l|l|l|l|l|l|l|l|}
\hline (36) & Án & ntá & qàwà & nà & lémíné & ké & kátú. & \\
\hline & & $2 S G$ & ST.NEG & devoir ${ }^{B}$ & INF & enfant & DEM & frapper & \\
\hline & \multicolumn{7}{|l}{} \\
\hline
\end{tabular}

\begin{tabular}{|l|l|l|l|l|l|l|l|l|l|}
\hline$(37)$ & Ké & sáhàré & nàn & qáxásá & ké & báanè & yí. & \\
\hline & DEM & médicament & ST & être_amer & DEM & un & POSTP & \\
\hline
\end{tabular}

cf. nèyê 'facile', nèwó 'devenir facile'

- ñérà 'être égal'

\begin{tabular}{|l|l|l|l|l|l|l|l|l|l|}
\hline & $(33)$ & À & sú & nàn & ñérà & yàxàré-n & màxá. & & \\
\hline & & $3 S G$ & tout & ST & être_égal & femme-D & pour & & \\
\hline \multicolumn{8}{|c|}{} \\
\hline
\end{tabular}

cf. ñérò 'devenir égal’ ${ }^{14}$

- yáríñá, yáriñányá 'être laid'

\begin{tabular}{|l|l|l|l|l|l|l|l|l|}
\hline & (34) & Túrúnyè-n & nàn & yáríná(nyá) & túunè-n & yá. & & \\
\hline & & hyène-D & ST & être_laid & chacal-D & POSTP & & \\
\hline & & 'L'hyène est plus laide que le chacal.' \\
\hline
\end{tabular}

cf. yáriñànyê 'laid', yáriñányó ‘devenir laid'

- qáwà 'se ressembler, aller bien ensemble'

\begin{tabular}{|l|l|l|l|l|l|l|l|l|}
\hline (35) & A & dò & $\hat{i}$ & yàqqê-n & ntá & qàwà. & \\
\hline & & $3 S G^{\mathrm{H}}$ & avec & REFL & épouse-D & ST.NEG & s'accorder $^{\mathrm{B}}$ & \\
\hline \\
\hline \multicolumn{8}{|c|}{ 'Lui et son épouse ne sont pas faits l'un pour l'autre.' } \\
\hline
\end{tabular}

cf. qáwò 'devenir semblable"15

- qáwà 'devoir’

- qáxásá 'être amer'

Ce verbe statif ne correspond à aucun adjectif ou verbe, mais il est probablement issu d'une dérive sémantique à partir de qáwà 'aller bien ensemble' - cf. (Creissels 2017). 


\begin{tabular}{|l|l|l|l|l|l|l|l|l|l|}
\hline & $(41)$ & Ké & háté & nàn & cáláxá & ké & báanè & yí. & \\
\hline & & DEM & peau & ST & être_lisse & DEM & un & POSTP & \\
\hline \multicolumn{8}{|c|}{} \\
\hline \multicolumn{1}{|c|}{ 'Cette peau est plus lisse que cette autre.' } \\
\hline
\end{tabular}


cf. sálàqqe 'lisse, visqueux', sáláxó 'devenir lisse, visqueux'

- sirì 'être bon'

\begin{tabular}{|l|l|l|l|l|l|l|l|l|}
\hline & (42) & Qàncó-n & nàn & círi & tí & lémúnù-n & yá. & \\
\hline & & bouillie-D & ST & être_bon & avec & enfant.PL & POSTP & \\
\hline & \multicolumn{6}{|c|}{ 'La bouillie de farine est bonne pour les enfants.' } \\
\hline
\end{tabular}

cf. sírè 'bon', síró 'devenir bon'

- síri 'devoir'

\begin{tabular}{|l|l|l|l|l|l|l|l|l|}
\hline & $(43)$ & $\delta$ & nàn & círì & dàgà-ná & kéetá. & & \\
\hline & & $1 \mathrm{PL}$ & ST & devoir & partir-GER & maintenant & & \\
\hline & & \multicolumn{6}{|c|}{ 'Nous devons partir maintenant.' } \\
\hline
\end{tabular}

Ce verbe statif ne correspond à aucun adjectif ou verbe, mais il est probablement issu d'une dérive sémantique à partir de síri 'être bon', selon un processus déjà évoqué ci-dessus pour son synonyme qáwà - cf. (Creissels 2017).

- táwó, táwú 'être chaud, rapide’

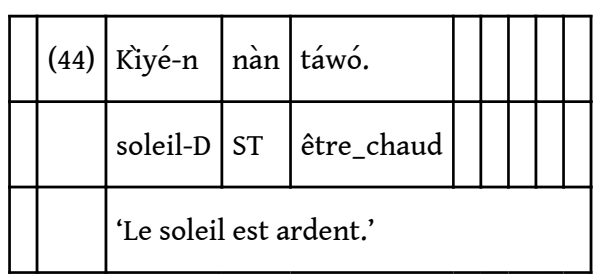

8 cf. táyè 'chaud', táwó 'devenir chaud'

- tintá 'être proche', cf. tintê 'proche', tìntó 's'approcher'

\section{Verbes statifs dérivés par suffixation}

Il existe en soninké une dérivation très productive d'adjectifs déverbaux au moyen d'un suffixe -nte: wáxà-ntê 'fou' < wáxà 'perdre la tête', bònò-ntê 'abîmé' < bònó 'abîmer', yáali-ntè 'brillant' < yáali 'briller', etc. Les adjectifs déverbaux de ce type ont une forme non-autonome terminée en a : wáxántá-, bònontà-, yáalintá-, etc. Par exemple, múxúntè 'caché' (< múxú 'cacher') apparaît à la forme non-autonome múxúntá- dans un composé comme múxúntá-n-gáagà 'acheter en cachette’.

Pour certains de ces adjectifs déverbaux au moins, il existe un verbe statif de même sens dont la forme coïncide (au moins segmentalement) avec la forme non-autonome de l'adjectif déverbal. Par exemple : 
Tableau 5 : Verbes, adjectifs déverbaux et verbes statifs

\begin{tabular}{|l|l|l|l|l|l|}
\hline verbe & & adjectif déverbal & \multicolumn{2}{|c|}{ verbe statif } \\
\hline bònó & 'abîmer' & $\begin{array}{l}\text { bònòntê } \\
\text { f.n.aut. bònòntà- }\end{array}$ & 'abîmé', 'nuisible' & bònòntá & 'être nuisible' \\
\hline yáali & 'briller' & $\begin{array}{l}\text { yáalintè } \\
\text { f.n.aut. yáalintá- }\end{array}$ & 'brillant' & yáalintá & 'être brillant' \\
\hline tóoxi & 'se fâcher' & $\begin{array}{l}\text { tóoxintè } \\
\text { f.n.aut. tóoxintá- }\end{array}$ & 'féroce' & tóoxintáa & 'être féroce' \\
\hline
\end{tabular}

\begin{tabular}{|l|l|l|l|l|l|l|l|}
\hline (45) & Hàabà-n-báanà-n & nàn & bònò-ntá & jàrinté-n & jà. & & \\
\hline & père-EP-un-D & ST & abîmer-DER & lion-D & POSTP & & \\
\hline
\end{tabular}

\begin{tabular}{|l|l|l|l|l|l|l|l|l|}
\hline (46) & Ké & hóróntó & nàn & tóoxí-ntá & ké & báané & yì. & \\
\hline & DEM & piment & ST & se_fâcher-DER & DEM & un & POSTP & \\
\hline \multicolumn{7}{|l}{} \\
'Ce piment-ci est plus fort que cet autre.' \\
\hline
\end{tabular}

\section{Les verbes statifs dans le système de dérivation du soninké}

102 Il ressort de l'inventaire donné à la section 5 qu'en règle générale, les verbes statifs sont en relation avec un adjectif de même sens, ainsi qu'avec un verbe qui signifie le processus aboutissant à l'état signifié par le verbe statif, et ces relations s'expriment essentiellement par une alternance de voyelles à la finale du lexème.

Pour les verbes statifs en relation avec un adjectif déverbal en -nte (cf. 5.2), la coïncidence entre le verbe statif et la forme non-autonome de l'adjectif déverbal autorise une description, soit en termes de dérivation parallèle (le même dérivatif ajouté à un verbe donnant naissance à un adjectif et à un verbe statif), soit en termes de conversion des adjectifs déverbaux en verbes statifs.

Pour les verbes statifs primaires, l'analyse de la relation avec les verbes et adjectifs correspondants demande tout d'abord de récapituler les correspondances attestées dans l'inventaire donné en 5.1 :

Tableau 6 : Verbes statifs, adjectifs et verbes intransitifs

\begin{tabular}{|l|l|l|l|l|}
\hline verbe statif & adjectif & & verbe intransitif \\
\hline
\end{tabular}




\begin{tabular}{|c|c|c|c|}
\hline báanà & 'être égal’ & báané & báanó \\
\hline báráxátá & 'être gros, fier' & báráxàtê & báráxátó \\
\hline dàrá & 'être gros, important' & dàrê & dàré \\
\hline dehá & 'être court, bas' & dèppê & dèhó \\
\hline dinká & 'être gros' & dinkâ & dinkó \\
\hline gàbá & 'être fréquent' & gàbê & gàbó \\
\hline giilá & 'être long, haut' & gìile & gìiló \\
\hline hàsú & 'être meilleur' & hàsê & hàsó \\
\hline húnyù & 'être supérieur' & - & húnyò \\
\hline jàwú & 'être rapide' & jàyê & jàwó \\
\hline láatú & 'être loin' & láatè & láató \\
\hline linná, lingí & 'être doux, agréable' & linjè & linjó \\
\hline loxá & 'être petit, peu' & loxe $\hat{e}$ & loxó \\
\hline misá & 'être mince, mesquin' & mise $\hat{e}$ & misó \\
\hline nèhá, nèwú & 'être facile' & nèyê & nèwó \\
\hline nérà & 'être égal' & - & ñérò \\
\hline yáríñá(nyá) & 'être laid' & yáriñànyê & yáriñányó \\
\hline qáwà & 'se ressembler' & - & qáwó \\
\hline qáwà & ‘devoir’ & - & - \\
\hline qáxásá & 'être amer' & qáxàccê & qáxásó \\
\hline qoorá & 'être grand' & qoorê & qoooró \\
\hline qótà, qótò & 'être dur, difficile, lourd' & qótè & qóto \\
\hline sáagá, sáagú & 'être courageux' & sáagè & sáagó \\
\hline sáláxá & 'être lisse, visqueux' & sálàqqê & sáláxó \\
\hline síri & 'être bon' & sírè & siró \\
\hline sirì & ‘devoir’ & - & - \\
\hline táwó, táwú & 'être chaud' & táyè & táwó \\
\hline
\end{tabular}




\begin{tabular}{|l|l|l|l|l|l|}
\hline tìntá & 'être proche' & tìntê & & tintó \\
\hline
\end{tabular}

La régularité dominante est très clairement que la terminaison $a$ pour le verbe statif correspond à une terminaison $e$ pour l'adjectif, et à une terminaison o pour le verbe intransitif. Il est toutefois frappant de constater que les verbes statifs présentent plus de variété dans leurs terminaisons que les adjectifs et les verbes intransitifs qui leur correspondent :

- parmi les adjectifs qui figurent dans la liste, un seul (dinkâ 'gros') présente une terminaison autre que $e$;

- parmi les verbes intransitifs qui figurent dans la liste, un seul (dàré 'devenir important') présente une terminaison autre que $o$, et encore faut-il noter que ce verbe peut être analysé comme le dérivé médiopassif régulier du verbe transitif dàró 'accorder de l'importance à' ;

- parmi les verbes statifs qui figurent dans la liste, quatre présentent une terminaison $u$, deux présentent une terminaison $i$, et cinq autres manifestent une hésitation entre la terminaison $a$ et une autre terminaison.

Compte tenu de ce qui se passe avec les verbes statifs dérivés par suffixation (qui, au moins segmentalement, sont homonymes de la forme non-autonome de l'adjectif déverbal correspondant), il convient de souligner que très peu de verbes statifs primaires sont homonymes de la forme non-autonome de l'adjectif correspondant. Les seuls cas nets dans les limites de mes données sont báanà 'être égal' (báané 'un' a pour forme non-autonome báaná-) et dinká 'être gros' (l'adjectif correspondant, dinkâ, a pour forme non-autonome dinkà-). Les hésitations dans la voyelle finale de certains verbes statifs ainsi que dans la voyelle finale de quelques adjectifs à la forme non-autonome rendent difficile une évaluation plus précise, mais parmi les adjectifs qui figurent dans cette liste, très peu m'ont été donnés avec la possibilité d'une forme non-autonome se terminant par une voyelle autre que $i$, alors que $i$ est très peu attesté comme terminaison possible de verbes statifs. La régularité dominante est l'alternance illustrée par giiile 'long', qui a pour forme non-autonome gìili- et correspond au verbe statif giiilá.

Avant de revenir à la question des verbes statifs, il est aussi utile de dire quelques mots sur la relation entre adjectifs en $e$ et verbes intransitifs en $o$, car cette relation vaut aussi pour beaucoup de couples adjectif / verbe sans verbe statif correspondant.

.M. Diagana (1995:123-125) a recensé une cinquantaine d'adjectifs primaires ${ }^{16}$ (c'est-à-dire non analysables comme le produit d'une dérivation par affixation), et ces données ont été confirmées par l'enquête lexicale que j'ai moi-même effectuée dans l'objectif de l'analyse phonologique du soninké du Kingi (Creissels 2016). Seuls cinq des adjectifs primaires (10\%) ne se terminent pas par $e$ : dinkâ 'énorme', dúurò 'vide', hákkà 'plein', kúrúnbà 'nouveau' et qàntô 'gros'.

109 Quant à la relation entre adjectifs en $e$ et verbes en 0 , O. M. Diagana (1995:260-264) l'analyse sous l'intitulé de "verbalisation adjectivale ", ce qui implique qu'il considère comme allant de soi que la relation doit être orientée dans le sens « adjectif > verbe ». Il ne fournit d'ailleurs aucune justification de ce choix, qui est pourtant tout sauf évident. 
110 Une observation importante est qu'il y a très peu d'exceptions à la règle selon laquelle à chaque adjectif primaire correspond un verbe dont la terminaison est $o$. Les exceptions sont les suivantes :

- les adjectifs de couleur binnê 'noir', dùnbê 'rouge' et qúllè 'blanc', qui correspondent aux verbes hìná 'noircir', dùnbú 'rougir' et qúrá 'blanchir' ;

- dúurò 'vide' et kúrúnbà 'nouveau', pour lesquels le verbe correspondant est formé au moyen d'un suffixe -aaxu qui est par ailleurs productif pour la formation de verbes à partir de noms : dúur-áaxú 'se vider', kúrúnb-áaxú 'renouveler'.

Ceci dit, l'hypothèse d'une dérivation orientée de l'adjectif vers le verbe se heurte toutefois à une difficulté importante du fait que dans pas mal de cas, l'adjectif est segmentalement plus complexe que le verbe, avec une gémination consonantique qui n'a pas d'équivalent pour le verbe correspondant (comme dans deppê 'court' / dehó 'devenir court').

112 Mais l'argument le plus fort contre l'hypothèse d'une dérivation orientée de l'adjectif vers le verbe est que seuls les adjectifs primaires correspondent à des verbes en 0 . Or s'il s'agissait réellement d'une dérivation adjectif > verbe, il n'y aurait aucune raison pour que les adjectifs dérivés par suffixation ne permettent pas eux aussi la formation de verbes en $o$.

113 En ce qui concerne maintenant le statut des verbes statifs primaires dans le système de dérivation du soninké, O. M. Diagana (1995:263-264) se contente d'évoquer une « verbalisation adjectivale en $a$ » qui se distingue de la "verbalisation adjectivale en $o$ » par le trait «+ intensif». Il n'envisage pas la possibilité que cette "verbalisation adjectivale " cible en fait une catégorie de lexèmes distincte de celle des verbes proprement dits. Il observe seulement en passant l'absence de valeur injonctive dans l'usage du marqueur prédicatif $n$ (an) avec les verbes statifs, en omettant d'ailleurs de mentionner le couplage avec le marqueur prédicatif négatif ntá, qui est pourtant essentiel.

114 Cette notion de "verbalisation adjectivale en $a$ » est problématique à double titre: d'abord la catégorie visée n'est pas celle des verbes proprement dits, et ensuite les formes supposées être le produit de cette dérivation sont loin de se terminer uniformément en $a$.

115 Compte tenu de ce qui est l'objectif central de cet article (attirer l'attention sur la nécessité de prendre en compte une catégorie de verbes statifs dans la systématique des parties du discours en soninké), je ne chercherai pas ici à proposer une solution définitive à la question de savoir comment analyser formellement la relation entre les adjectifs et les verbes en o qui leur correspondent. Il me semble prudent de s'en tenir au moins provisoirement à l'idée que, à l'exception de qáwà 'devoir' et síri 'devoir' (qui d'un point de vue strictement synchronique ne sont apparentés ni à un verbe, ni à un adjectif), les verbes statifs sont issus de radicaux abstraits au statut catégoriel sous-spécifié, qui par dérivation équipollente donnent naissance, soit à des dyades «verbe statif / verbe intransitif» (comme par exemple húngù 'être supérieur' / húngò 'dépasser'), soit à des triades "verbe statif / adjectif / verbe intransitif » (comme síri 'être bon' / sirè 'bon' / siró 'devenir bon'), tandis que d'autres radicaux abstraits au statut catégoriel sous-spécifié donnent naissance par dérivation équipollente à des dyades «adjectif / verbe intransitif » (comme par exemple sénnè 'pur, propre' / sénó 'devenir pur, propre'). 


\section{Conclusion}

Dans cet article, j'ai montré que le système des parties du discours en soninké (au moins dans la variété du Kingi) comporte une catégorie de verbes statifs dont les caractéristiques morphosyntaxiques sont à bien des égards semblables à celles des verbes statifs des langues mandingues. Le point essentiel est la compatibilité de ces lexèmes avec un couple unique de marqueurs prédicatifs, le couplage des deux marqueurs prédicatifs en question étant différent de ceux qu'ils forment dans le cadre de prédication verbale. Une différence importante avec les langues mandingues est toutefois le statut des verbes statifs dans le système de dérivation, car les triades " verbe statif / adjectif / verbe » mettant en jeu une simple alternance de la voyelle finale des lexèmes, qui constituent la règle générale en soninké, n'ont pas d'équivalent dans les langues mandingues.

117 Il resterait maintenant à effectuer sur d'autres variétés de la langue soninké les enquêtes permettant de déterminer jusqu'à quel point la description proposée ici est représentative de ce qui existe dans les autres variétés.

\section{Abréviations et gloses}

\begin{tabular}{|l|l|}
\hline ACP & accompli \\
\hline ANTIP & antipassif \\
\hline B & ton grammatical bas substitué au schème tonal lexical (verbes, noms et numéraux) \\
\hline BH & ton grammatical bas-haut substitué au schème tonal lexical (noms) \\
\hline CAUS & causatif \\
\hline COPEQ & copule équative \\
\hline COPLOC & copule de localisation \\
\hline D & marqueur de détermination nominale \\
\hline DEM & démonstratif \\
\hline DER & dérivatif \\
\hline EP & nasale épenthétique \\
\hline FOC & focalisateur \\
\hline GER & gérondif \\
\hline H & ton grammatical haut (pronoms de troisième personne) \\
\hline INACP & inaccompli \\
\hline
\end{tabular}




\begin{tabular}{|l|l|}
\hline INF & infinitif \\
\hline INTR & intransitif \\
\hline NEG & négatif \\
\hline NMLS & nominalisation \\
\hline PL & pluriel \\
\hline POSTP & postposition multifonction \\
\hline Q & marque de question totale \\
\hline REFL & réfléchi \\
\hline SG & singulier \\
\hline ST & marqueur prédicatif des verbes statifs \\
\hline SUBJ & subjonctif \\
\hline SUJ & marque de la fonction sujet pour les interrogatifs et les groupes nominaux focalisés \\
\hline TR & marqueur de transitivité \\
\hline
\end{tabular}

\section{BIBLIOGRAPHIE}

\section{Références bibliographiques}

Creissels, Denis. 1985. Les verbes statifs dans les parlers manding. Mandenkan 10. 1-32.

Creissels, Denis. 2016. Phonologie segmentale et tonale du soninké (parler du Kingi). Mandenkan 55. 3-174.

Creissels, Denis. 2017. Similarity, suitability, and non-epistemic modalities (volitionality, ability, and obligation). In Yvonne Treis \& Martine Vanhove (eds.), Similative and equative constructions: A cross-linguistic perspective, 79-89. John Benjamins.

Diagana, Ousmane Moussa. 1995. La langue soninkée : morphosyntaxe et sens à travers le parler de Kaédi (Mauritanie). Paris : Harmattan.

Diagana, Ousmane Moussa. 2011. Dictionnaire soninké-français (Mauritanie). Paris : Karthala.

Diagana, Yacouba. 1994. Éléments de grammaire du soninké. 2 vols. Paris : Les documents de Linguistique africaine. 
Dumestre, Gérard. 2003. Grammaire fondamentale du bambara. Paris : Karthala.

Smeltzer, Brad \& Susan Smeltzer. 2001. Lexique Soninké-Français-Anglais. Soninke-French-English Dictionary. Index Français-Soninké, English-Soninke. Bamako.

Vydrine, Valentin. 1990. Les adjectifs prédicatifs en bamana. Mandenkan 20. 47-90.

Vydrine, Valentin. 1999. Les parties du discours en bambara : un essai de bilan. Mandenkan 35. 7393.

\section{NOTES}

1. Sur les verbes statifs des langues mandingues, cf. (Creissels 1985 ; Dumestre 2003:169-178 ; Vydrine 1990 ; Vydrine 1999).

2. Dans cet exemple, du point de vue segmental, la relation entre le singulier et la forme non-autonome se résume à un changement de voyelle finale $(i \sim e)$ et il en va de même pour la relation entre le singulier et le pluriel $(e \sim u)$. Toutefois, pour certains noms, la forme non-autonome se déduit de la forme du singulier par la suppression de la voyelle finale et le remplacement de la consonne précédente ou du groupe consonantique précédent par une nasale (comme dans sámáqqè f.n.aut. sámán- 'serpent'), ce qui s'explique par le fait qu'en soninké, la position de coda peut seulement être occupée par une nasale sous-spécifiée ou par une copie de l'attaque de la syllabe suivante. Quant au pluriel, une partie des noms le marquent par l'adjonction de -nú (ou -ní) à la forme du singulier.

3. La règle est que la forme non-autonome a un schème tonal entièrement $\mathrm{B}$ ou entièrement $\mathrm{H}$ selon que le ton initial de la forme de singulier est $\mathrm{B}$ ou $\mathrm{H}$.

4. $\mathrm{S}=$ sujet, $\mathrm{mp}=$ marqueur prédicatif, $\mathrm{O}=$ objet, $\mathrm{V}=$ verbe, $\mathrm{X}=$ oblique.

5. Les verbes qui figurent dans ce tableau illustrent la formation régulière du gérondif, par adjonction d'un suffixe $-n V$, mais un certain nombre de verbes dissyllabiques avec une consonne simple en position médiane font leur gérondif par une transformation de la consonne médiane, qui devient dans tous les cas géminée, accompagnée parfois de modifications des voyelles qui ont un caractère irrégulier (par exemple búgú 'sortir' a pour gérondif bákká). Sur la formation du gérondif, cf. (Creissels 2016).

6. Le projectif (terme emprunté à Yacouba Diagana, qui caractérise ce tiroir verbal comme signifiant «un projet dont la réalisation est souhaitée, voire exigée ») peut être vu sémantiquement comme une variété de futur. Ce tiroir s'utilise notamment pour donner des instructions à un interlocuteur, et il a aussi un emploi optatif (comme dans Hári ná án kísi ! 'Que Dieu te sauve !').

7. Le subjonctif s'utilise en phrase indépendante pour formuler des injonctions concernant d'autres personnes que l'interlocuteur ou les interlocuteurs. Les deux variantes du marqueur du subjonctif dans la construction intransitive (nàn et ' $n$ ) sont en variation libre. Enfin, on doit noter que les mêmes marqueurs figurent dans la construction infinitivale, qui diffère de la construction prédicative verbale par l'absence de groupe nominal précédent le marqueur prédicatif.

8. A l'impératif positif transitif, on a $\emptyset$ au singulier (la position de sujet restant vide) mais dà au pluriel, où la position de sujet est occupée par la variante qà du pronom de 2ème personne du pluriel qá. La forme tonale du pronom assure alors la distinction avec la 2ème personne du pluriel de l'accompli positif transitif. Par exemple, Qá dà dòrokén bòoxó, où qá a son ton inhérent $\mathrm{H}$, ne peut s'interpréter que comme une phrase assertive à l'accompli ('Vous avez déchiré le boubou'), tandis que Qà dà dòòkén bòoxó, où un ton $\mathrm{B}$ s'est substitué au ton $\mathrm{H}$ de qá, ne peut s'interpréter que comme une phrase impérative ('Déchirez le boubou !'), 
9. L'inaccompli positif 1 s'emploie dans une phrase ne comportant ni interrogatif, ni groupe nominal marqué comme focalisé, tandis que l'inaccompli positif 2 implique la présence d'un interrogatif ou d'un groupe nominal focalisé.

10. Háyí, forme grammaticalisée de l'impératif du verbe háyíi 'regarder', peut par ailleurs exprimer une valeur ostensive en se combinant simplement à un constituant nominal de la même façon que 'voici' en français : Múusá háyí ! 'Voici Moussa !' (litt. 'Regarde Moussa !').

11. Le même phénomène s'observe lorsqu'un constituant nominal en fonction de sujet ou d'objet dans la construction prédicative verbale est suivi d'un adjectif en fonction de prédicat second.

12. Pour la même raison, certains des verbes statifs qui figurent dans la liste lexicale donnée en annexe de (Creissels 2016) n'ont pas été repris ici.

13. Il n'y a pas d'adjectif correspondant à ce verbe statif.

14. Il n'y a pas d'adjectif correspondant à ce verbe statif.

15. Il n'y a pas d'adjectif correspondant à ce verbe statif.

16. Le terme utilisé par O. M. Diagana lui-même est " qualifiant pur ».

\section{RÉSUMÉS}

Cet article décrit une catégorie de mots du soninké jusqu'ici négligée dans les descriptions de cette langue. Les caractéristiques générales de cette catégorie sont comparables à celles des «verbes statifs » des langues mandingues. En particulier, ils assument la fonction prédicative en combinaison avec un couple unique de marqueurs prédicatifs (positif nàn vs. négatif ntá) qui sont attestés par ailleurs, mais appariés différemment, et avec des valeurs aspecto-modales dont ils sont dépourvus dans leur emploi avec les verbes statifs.

This article describes a lexical category found in Soninke that has been neglected so far. The general characteristics of this category are similar to those of the "stative verbs" found in Manding languages. In particular, they fulfill the predicative function in combination with a single pair of predicative markers (positive nàn vs. negative ntá) also attested with the other verbs, but in different pairings, and with aspecto-modal values of which they are deprived in their use with stative verbs.

В СТАТЬЕ РАССМАТРИВАЕТСЯ ЛЕКСИКОГРАММАТИЧЕСКИЙ КЛАСС СОНИНКЕ, КОТОРОМУ ДО СИХ ПОР УДЕЛЯЛОСЬ МАЛО ВНИМАНИЯ В ОПИСАНИЯХ ЭТОГО ЯЗЫКА. В ЦЕЛОМ ЭТОТ КЛАСС ЛЕКСЕМ БЛИЗОК ПО СВОИМ ХАРАКТЕРИСТИКАМ К «СТАТИВНЫМ ГЛАГОЛАМ» ЯЗЫКОВ МАНДЕН. В ЧАСТНОСТИ, СТАТИВНЫЕ ГЛАГОЛЫ В СОНИНКЕ ВЫСТУПАЮТ В ПРЕДИКАТИВНОЙ ФУНКЦИИ В СОЧЕТАНИИ ЛИШЬ С ОДНОЙ ПАРОЙ ПРЕДИКАТИВНЫХ ПОКАЗАТЕЛЕЙ (УТВЕРДИТЕЛЬНЫМ $n a$ И ОТРИЦАТЕЛЬНЫМ nta ПАРНОМ СООТНОШЕНИИ, И В ПОСЛЕДНЕМ СЛУЧАЕ ОНИ ВЫРАЖАЮТ НЕ ТЕ ЖЕ САМЫЕ АСПЕКТО-МОДАЛЬНЫЕ ЗНАЧЕНИЯ, ЧТО ПРИ СТАТИВНЫХ ГЛАГОЛАХ. 
INDEX

motsclesru ЯЗЫКИ МАНДЕ, СОНИНКЕ, ЧАСТИ РЕЧИ, СТАТИВНЫЕ ГЛАГОЛЫ

Mots-clés : mandé, soninké, parties du discours, verbes statifs

Keywords : Mande, Soninke, parts of speech, stative verbs

\section{AUTEUR}

\section{DENIS CREISSELS}

ДЕНИ КРЕСЕЛЬ

Université Lyon 2

denis.creissels@univ-lyon2.fr

http://deniscreissels.fr 